PNU-NTG-11/2004

\title{
Is there a crystalline state of nuclear matter?
}

\author{
U.T. Yakhshiev, ${ }^{1,2, \circledast ~ M . M . ~ M u s a k h a n o v, ~}{ }^{1,2, \oplus}$ and H.-Ch. Kim ${ }^{1, \oplus}$ \\ ${ }^{1}$ Department of Physics and Nuclear Physics 8 Radiation Technology Institute (NuRI), \\ Pusan National University, 609-735 Busan, Republic of Korea \\ ${ }^{2}$ Theoretical Physics Department 6 Institute of Applied Physics \\ National University of Uzbekistan, Tashkent-174, Uzbekistan
}

(Dated: March 2005)

\begin{abstract}
A possibility of the crystalline state of nuclear matter is discussed in a medium-modified Skyrme model. The interaction energy per nucleon in nuclear matter is evaluated by taking into account the medium influence on single nucleon-skyrmion properties and the tensor part of the nucleonnucleon potential, and by using a variational method of Hartree-Fock type including zero-point quantum fluctuations. It is shown that in this approach the ground state of nuclear matter has no crystalline structure due to quantum fluctuations as well as medium modifications of hadron properties.
\end{abstract}

PACS numbers: 12.39.Dc, 12.39.Fe, 21.65.+f, 21.30.Fe

Keywords: Nuclear matter, Crystalline structure, Medium modifications, Skyrme model

*Electronic address: yakhshiev@pusan.ac.kr

$\dagger$ Electronic address: musakhanov@pusan.ac.kr

‡Electronic address: hchkim@pusan.ac.kr 
1. After a pioneering work by Kutchera et al. 1] related to the dense-matter properties in the Skyrme model, Klebanov [2] discussed a possible formation of the Skyrmionic matter with the simple cubic crystalline structure due to tensor forces between the nucleon of a lattice-point and the nearest six nucleons of the unit cell. There has been a great amount of work on energetically favorable crystalline structures 3, 4, 5] and parallel studies of the Skyrme model on the hypersphere [6, 7, 8]. Former studies are still under discussion [9] and have concentrated on investigating the classical field configuration, assuming face centered cubic (FCC) structure. Obviously, one should quantize the model to investigate the real nuclear matter, but it is not at all easy to do it as explained in Ref. [10].

On the other hand, quantum fluctuations around a classical ground state can play an important role in leading to nuclear matter which is different in its behavior from the classical Skyrmion matter. A standard variational method of Hartree-Fock type used in Ref. [11] provides a theoretical framework to make it tractable to deal with nuclear matter with quantum fluctuations. Taking into account only the tensor part of the nucleon-nucleon (NN) interaction based on the one-pion-exchange potential (OPEP) and assuming the FCC structure, Diakonov and Mirlin [11] showed that at densities which are several times higher that that of ordinary nuclear matter a condensed state of nuclear matter may be still energetically favorable. Alternatively, using time-dependent numerical calculations on a quantized simple cubic lattice, Walhout [12] evaluated the zero-point kinetic energy of nucleons and found it to unbind the classical crystalline ground state. Consequently, one can conclude that due to the increasing number of neighboring nucleons the FCC structure appears in a deeper bound state.

However, above-mentioned studies did not consider possible medium modifications of single nucleon and pion properties and the corresponding medium-modified NN interaction [13, 14, 15, 16]. Refs. [14, 15, 16] considered the nucleon as a Skyrmion and then studied the influence of baryonic matter on its properties phenomenologically via medium modifications of pion fields. The results of these studies were in a qualitative agreement with experimental indications, e.g. the swelling of the nucleon in nuclear medium and the decrease of its mass. It was also shown that due to the influence of surrounding nuclear environment the tensor part of the NN potential is decreased [14]. The changes in hadron static properties and form factors of meson-nucleon vertices also lead to the suppression of the one-boson-exchange potential [15]. In this context, it is natural to ask and answer the question: How do these changes play a role in describing nuclear matter?

Since it is of great difficulty to quantize the Skyrmionic matter itself [10], we follow the phenomenological and variational method put forward by Ref. 11] in order to investigate how the medium modifications of the NN potential and of hadronic properties change the groundstate energy of nuclear matter and reestimate the role of zero-point quantum fluctuations around the minimum.

2. In order to calculate the medium-modifications of single hadron properties, we start with the effective chiral Lagrangian for the medium-modified Skyrmion [14]. Using the phenomenological values $b_{0}=b_{0}^{\text {phen }}=-0.024 m_{\pi}^{-1}, c_{0}=c_{0}^{\text {phen }}=0.15 m_{\pi}^{-3}$ of the effective pion-nucleon scattering lengths [17], one gets the density dependence of hadron properties as listed in Table $\square^{1}$ In the present work, the asterisk in notation indicates medium-

${ }^{1}$ We refer the reader to the works [14, 15], where the medium modifications of hadronic properties have 


\begin{tabular}{l|c|ccc|ccc}
\hline \hline \multicolumn{1}{c|}{$\rho / \rho_{0}$} & 0 & \multicolumn{3}{|c|}{0.5} & \multicolumn{3}{|c}{1.0} \\
\hline \multicolumn{1}{c|}{$g^{\prime}$} & - & 0.33 & 0.6 & 1 & 0.33 & 0.6 & 1 \\
\hline$g_{\pi N N}^{*}$ & 12.49 & 9.48 & 9.76 & 10.08 & 6.83 & 7.75 & 8.66 \\
$M_{N}^{*}[\mathrm{MeV}]$ & 868 & 743 & 756 & 770 & 635 & 675 & 715 \\
$m_{\pi}^{*}[\mathrm{MeV}]$ & 140 & 146 & 146 & 146 & 152 & 152 & 152 \\
$\Lambda^{*}[\mathrm{MeV}]$ & 528 & 484 & 489 & 494 & 448 & 462 & 477 \\
$M_{\Delta N}^{*}[\mathrm{MeV}]$ & 243 & 211 & 214 & 218 & 186 & 196 & 206 \\
\hline \hline
\end{tabular}

TABLE I: Comparison of hadronic properties in free space with those in nuclear matter with density $\rho$. In the second column the corresponding values in free space are listed, $g^{\prime}$ stands for the correlation parameter, $\rho_{0}=0.17 \mathrm{fm}^{-3}$ is the density of normal nuclear matter. $M_{\Delta N}^{*} \equiv M_{\Delta}^{*}-M_{N}^{*}$ is the $\Delta-\mathrm{N}$ mass splitting.

modified quantities. The results are presented for three different values of the LorentzLorenz parameter: the first and the second ones, i.e. $g^{\prime}=0.33$ and $g^{\prime}=0.6$, correspond to the classical and phenomenological values [17], respectively, and the third one is simply taken as $g^{\prime}=1$. One can see that hadronic properties as well as the pion-nucleon coupling constant are changed due to the presence of nuclear medium. Consequently, the nuclearmatter properties, being calculated by using the NN potential between the nearest neighbors in nuclear matter, are expected to be modified accordingly.

In order to study a phase structure of nuclear matter, we follow closely a variational procedure described in Ref. [11]. We define the position vector of an FCC lattice point $n$ by $\boldsymbol{r}_{0 n}$ and the optimum orientation (the relative orientation matrix in internal space) of the nucleon at this lattice point by $A_{0 n}$. The general orientation matrix $A$ is related to an arbitrary position vector in isospin space. The trial wave function of the crystal must be chosen as an antisymmetrized product of single-lattice-point wave functions $\psi(\boldsymbol{r}-$ $\left.\boldsymbol{r}_{0 n}, A^{+} A_{0 n}\right)$ localized near the lattice sites and the corresponding optimum orientations.

The overlap integrals of the non-nearest neighbors being neglected, the energy functional (interaction energy per nucleon) can be written as a following Hartree-Fock form:

$$
\begin{aligned}
& E^{*}=\int d^{3} r d A \psi^{*}\left(\boldsymbol{r}-\boldsymbol{r}_{0}, A^{+} A_{0}\right)\left(-\frac{1}{2 M_{N}^{*}} \frac{\partial^{2}}{\partial \boldsymbol{r}^{2}}+\frac{\boldsymbol{T}_{A}^{2}}{2 I^{*}}\right) \psi\left(\boldsymbol{r}-\boldsymbol{r}_{0}, A^{+} A_{0}\right) \\
& +\frac{1}{2} \sum_{n=1}^{12} \int d^{3} r d^{3} r^{\prime} d A d A^{\prime} \psi^{*}\left(\boldsymbol{r}-\boldsymbol{r}_{0}, A^{+} A_{0}\right) \psi^{*}\left(\boldsymbol{r}^{\prime}-\boldsymbol{r}_{0 n}, A^{\prime+} A_{0 n}\right) V^{*}\left(\boldsymbol{r}-\boldsymbol{r}^{\prime}, A^{+} A^{\prime}\right) \\
& \times\left[\psi\left(\boldsymbol{r}-\boldsymbol{r}_{0}, A^{+} A_{0}\right) \psi\left(\boldsymbol{r}^{\prime}-\boldsymbol{r}_{0 n}, A^{\prime+} A_{0 n}\right)-\psi\left(\boldsymbol{r}-\boldsymbol{r}_{0 n}, A^{+} A_{0 n}\right) \psi\left(\boldsymbol{r}^{\prime}-\boldsymbol{r}_{0}, A^{\prime+} A_{0}\right)\right]
\end{aligned}
$$

where $I^{*}$ denotes the moment of inertia of the Skyrmion in medium. This functional must be minimized with respect to the single-lattice-point wave function $\psi$ for a given distance $R=\left|\boldsymbol{r}_{0 n}-\boldsymbol{r}_{0}\right|$ between the nearest neighbors. It is clear that in general the quantities, $M_{N}^{*}, I^{*}, V^{*}$, are $R$-dependent, while the $R$ is related directly to the nuclear-matter density.

been investigated and inclusion of medium parameters $\left(b_{0}, c_{0}\right.$ and $\left.g^{\prime}\right)$ were discussed. 
We use the single-lattice-point trial function as follows:

$$
\begin{array}{r}
\psi\left(\boldsymbol{r}-\boldsymbol{r}_{0}, A^{+} A_{0}\right)=\varphi\left(\boldsymbol{r}-\boldsymbol{r}_{0}\right) \frac{D_{k k}^{1 / 2}\left(A^{+} A_{0}\right)+\alpha D_{k k}^{3 / 2}\left(A^{+} A_{0}\right)}{\sqrt{1+\alpha^{2}}}, \\
\varphi(\boldsymbol{r})=\left(\varkappa^{2} / \pi\right)^{3 / 4}\left(1+3 \eta+15 \eta^{2} / 4\right)^{-1 / 2} \exp \left\{-\varkappa^{2} r^{2} / 2\right\}\left(1+\eta \varkappa^{2} r^{2}\right),
\end{array}
$$

where $\varkappa$ measures the spatial extent of the wave function, $\alpha$ denotes the admixture of the $\Delta$ resonance, $D_{a b}^{T}(A)$ is an $\mathrm{SU}(2)$ Wigner $D$ function, and $\eta$ stands for an additional variational parameter. Putting $\eta=0$, one can get the corresponding trial function similar to Ref. [11].

In general, NN potential can be presented in the following form:

$$
V^{*}\left(\boldsymbol{R}, A_{1}^{+} A_{2}\right)=V_{1}^{*}(R)+\frac{1}{2} \operatorname{Tr}\left(A_{1}^{+} A_{2} \sigma_{i} A_{2}^{+} A_{1} \sigma_{j}\right)\left[\left(3 \hat{R}_{i} \hat{R}_{j}-\delta_{i j}\right) V_{2}^{*}(R)+\delta_{i j} V_{3}^{*}(R)\right],
$$

where $\hat{R}$ is the corresponding unit vector along the line joining the centers of two nucleons and $A_{1}^{+} A_{2}$ denotes their relative orientation matrix in internal space. Utilizing Eqs. (2) -(3) of the lattice-point trial wave function and the NN potential given in the form of Eq. (4), one can arrive at the final expression for the energy functional (Eq. (11)):

$$
\begin{aligned}
E^{*}= & \frac{\alpha^{2}}{1+\alpha^{2}} M_{\Delta N}^{*}+\left(1+3 \eta+\frac{15 \eta^{2}}{4}\right)^{-1}\left(1+\eta+\frac{11}{4} \eta^{2}\right) \frac{3 \varkappa^{2}}{4 M_{N}^{*}}+E_{H}^{*}+E_{F}^{*}, \\
E_{H}^{*}= & \frac{3}{\pi^{2}}\left(1+3 \eta+\frac{15 \eta^{2}}{4}\right)^{-2} \int_{0}^{\infty} d q q^{2} \exp \left\{-\frac{q^{2}}{2 \varkappa^{2}}\right\} \\
& \times a^{2}\left[V_{1}^{*}(q)+\frac{(1+\alpha)^{2}}{9\left(1+\alpha^{2}\right)^{2}}\left(\frac{V_{2}^{*}(q)}{q^{2}} \frac{\partial^{2}}{\partial R^{2}}-V_{3}^{*}(q)\right)\right] \frac{\sin q R}{q R}, \\
E_{F}^{*}= & -\frac{3}{\pi^{2}}\left(1+3 \eta+\frac{15 \eta^{2}}{4}\right)^{-2}\left[\frac{4\left(1-\alpha-\alpha^{2}\right)}{3\left(1+\alpha^{2}\right)}\right]_{0}^{2} \int_{0}^{\infty} d q q^{2} \exp \left\{-\frac{\varkappa^{2} R^{2}}{2}-\frac{q^{2}}{2 \varkappa^{2}}\right\} \\
& \times\left[\left(\frac{b^{2}}{3}+\frac{4 b c}{15}+\frac{3 c^{2}}{35}\right) V_{2}^{*}(q)+\left(b^{2}+\frac{2 b c}{3}+\frac{c^{2}}{5}\right) V_{3}^{*}(q)\right],
\end{aligned}
$$

where the variables $a, b$, and $c$ are defined as

$$
\begin{aligned}
a & \equiv\left[1+\left(6-q^{2} / \varkappa^{2}\right) \eta / 2+\left(60-20 q^{2} / \varkappa^{2}+q^{4} / \varkappa^{4}\right) \eta^{2} / 16\right], \\
b & \equiv a+R^{2} \varkappa^{2}\left[\eta / 2+\left(4+R^{2} \varkappa^{2}-2 q^{2} / \varkappa^{2}\right) \eta^{2} / 16\right] \\
c & \equiv 4 q^{2} R^{2} \eta^{2} / 16 .
\end{aligned}
$$

To compute the ground-state energy in nuclear matter, we keep only a tensor part of the potential in Eq.(41), putting $V_{1}^{*}=V_{3}^{*}=0$. This tensor potential arises from one-pion exchange, which is expressed in momentum space as follows:

$$
V_{2}^{*}(q)=-\left(\frac{3 g_{\pi N N}^{*}}{2 M_{N}^{*}}\right)^{2} \frac{\boldsymbol{q}^{2}}{\boldsymbol{q}^{2}+m_{\pi}^{* 2}} \frac{\Lambda^{* 2}}{\boldsymbol{q}^{2}+\Lambda^{* 2}} .
$$

The effective values of the potential parameters and their density dependence can be calculated by using the medium-modified Skyrme Lagrangian given in Ref. [14] $]^{2}$. We present

\footnotetext{
${ }^{2}$ The medium-modified values of the OPEP parameters at some densities are presented in Table \where $g_{\pi N N}^{*}$ and $\Lambda^{*}$ are extracted from the medium-modified $\pi N N$ form factor (see Ref. 15] for more details).
} 
two different sets of OPEP parameters in free space: OPEP I $\left(g_{\pi N N}=14, M_{N}=940 \mathrm{MeV}\right.$, $m_{\pi}=140 \mathrm{MeV}, \Lambda=500 \mathrm{MeV}, M_{\Delta N}=300 \mathrm{MeV}$ ) used in Ref. 11] and OPEP II which coincides with the second $(\rho=0)$ column of Table I. We emphasize that our aim is not to describe quantitatively the NN potential in free space but to study qualitative changes in interaction energy due to the medium modifications.

These two parameter sets show almost the same dependence on $R$ for the interaction energy per nucleon as illustrated in Fig. 1. The curve with asterisks which corresponds to the OPEP I set with $\eta=0$ is equivalent with that of Ref. [11]. The curve with stars corresponding to the OPEP II with $\eta=0$ draws the result of the present calculation. Though we are not able to distinguish the results with the OPEP II from those with the OPEP I in shorter ranges, they start to get deviated each other when $R$ grows: The $\pi N N$ coupling constant in the OPEP I set is larger than that in the OPEP II, so that the interaction energy becomes more attractive. Note that the solutions with the OPEP II $(\eta \neq 0)$ are stable with respect to the change of the lattice-point nucleon trial functions. The additional parameter $\eta$ plays an important role only at larger distances, i.e. $R \geq 1.6 \mathrm{fm}$, where the energy is getting lower.

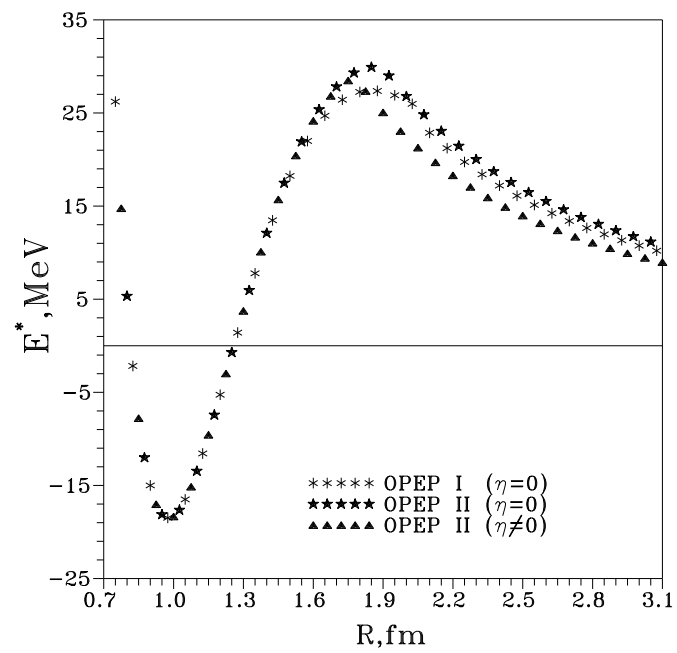

FIG. 1: The minimization of the interaction energy $E^{*}$ (Eq. (5) ) by using two sets of the OPEP parameters. The OPEP I $(\eta=0)$ corresponds to the results of Ref. 11]. Those with the OPEP II are drawn for two different values of the additional variational parameter, which is defined in Eq. (3), i.e. $\eta=0$ and $\eta \neq 0$, respectively.

In order to see how the ground-state energy of nuclear matter is changed with the medium modifications of the potential parameters taken into account, we begin with the OPEP II for both $\eta=0$ and $\eta \neq 0$ cases so that we can keep our investigation in a self-consistent manner. The density of the medium in the FCC structure is defined as $\rho=\sqrt{2} / R^{3}$ [1]]. The normal nuclear-matter density $\rho=0.17 \mathrm{fm}^{-3}$ corresponds to a distance between the nearest neighbors: $R \approx 2.03 \mathrm{fm}$. The medium modifications of the single hadron properties are expressed via three phenomenological quantities such as $b_{0}, c_{0}, g^{\prime}$ for a given density of nuclear matter [14]. We now treat the phenomenological effective $S$ - and $P$-wave pionnucleon scattering lengths $b_{0}, c_{0}$, and the correlation parameter $g^{\prime}$ in deriving the interaction energy per nucleon, respectively. While $g^{\prime}$ is fixed like $g^{\prime}=1$, we can examine how the inter- 


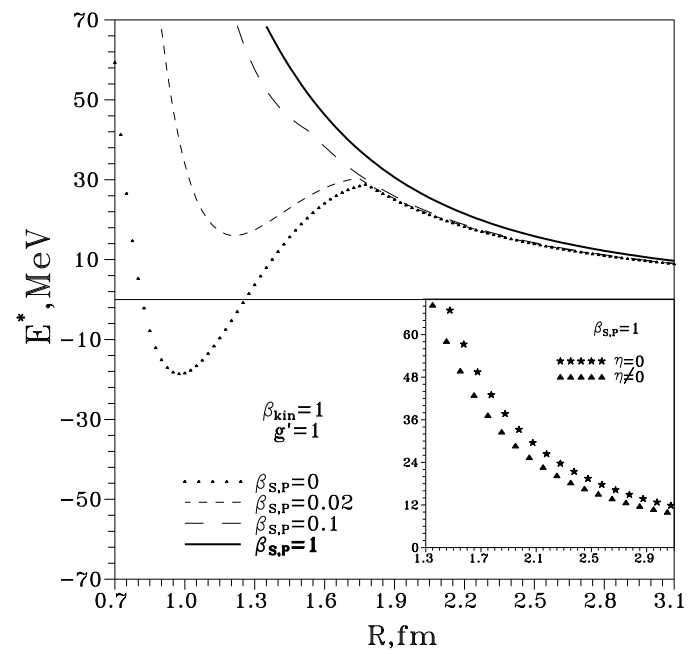

FIG. 2: The effect of the medium modifications of hadron properties on the interaction energy per nucleon. The dotted curve represents the case, where the medium modifications of hadron properties are not taken into account $\left(\beta_{S, P}=0\right)$, while the short-dashed one depicts the case when we consider the empirical parameters $b_{0}$ and $c_{0}$ by $2 \%$, i.e. $\beta_{S, P}=0.02$. The long-dashed curve draws the result with $\beta_{S, P}=0.1$, whereas the solid one takes into account $\beta_{S, P}=1$, i.e. the full effect of the medium modifications. In these curves, we always consider $\eta \neq 0$. In the small panel, two different curves are shown in the case of $\beta_{S, P}=1$, i.e. $\eta=0$ and $\eta \neq 0$, respectively.

action energy per nucleon depends on the parameters $b_{0}$ and $c_{0}$. Introducing the controlling variables $\beta_{S}$ and $\beta_{P}$, we can rewrite $b_{0}$ and $c_{0}$ as follows:

$$
b_{0}=b_{0}^{\text {phen }} \beta_{S}, \quad c_{0}=c_{0}^{\text {phen }} \beta_{P} .
$$

They allow us to include the medium effects in a controlled manner: $\beta_{S, P}$ are varied in the range of $0 \leq \beta_{S, P} \leq 1$. Putting $\beta_{S}=\beta_{P}=0$, we restore them in free space.

Similarly, we introduce the overall kinetic factor $\beta_{k i n} \leq 1$ in the energy functional (5) to show the importance of zero-point kinetic and iso-rotational ( $\Delta$ admixture) fluctuations:

$$
E^{*}=\left[\frac{\alpha^{2}}{1+\alpha^{2}} M_{\Delta N}^{*}+\left(1+3 \eta+\frac{15 \eta^{2}}{4}\right)^{-1}\left(1+\eta+\frac{11}{4} \eta^{2}\right) \frac{3 \varkappa^{2}}{4 M_{N}^{*}}\right] \beta_{k i n}+E_{H}^{*}+E_{F}^{*} .
$$

3. The effect of the medium parameters $b_{0}, c_{0}$ on the interaction energy per nucleon (Eqs. (5) , (91)) is depicted in Fig. 2. If the medium modifications are ignored, the quantum fluctuations are not enough to loose a close-packed FCC structure. Thus, nuclear matter can be formed as a FCC crystal when the density is several times higher than that of normal nuclear matter [11], as shown in the dotted curve $\left(\beta_{S, P}=0\right)$ in Fig. 2. With $\beta_{S, P}$ switched on, one can see that the corresponding modifications of the NN tensor potential essentially changes this picture of nuclear matter. It implies that including a small amount of the medium modifications breaks the condensed (or solid) FCC structure, as presented in short and long dashed curves in Fig. 2. It is found that $\beta_{S, P}=0.1$ breaks already the FCC 
structure. Consequently, the full consideration of the medium modifications, i.e. $\beta_{S, P}=1$ does not allow nuclear matter to be found in the form of a FCC crystal. This result is drawn in the solid curve in Fig. 2 and stable under the change of $\eta$, which is shown in the small panel of Fig. 2,

The effect of the Lorentz-Lorenz parameter on the interaction energy per nucleon is drawn in Fig. 3. We present here the results with quantum fluctuations and medium modification

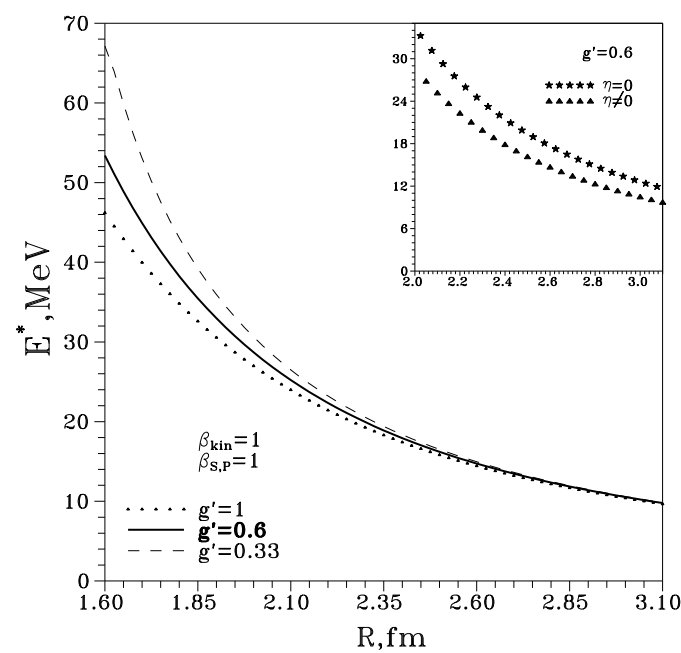

FIG. 3: The effect of the Lorentz-Lorenz parameter $g^{\prime}$ on the interaction energy per nucleon. Dotted, solid, and dashed curves correspond to the results for $g^{\prime}=1, g^{\prime}=0.6$ (phenomenological value), and $g^{\prime}=0.33$ (classical value), respectively. The medium modifications of the OPEP parameters are fully taken into account, i.e. $\beta_{S, P}=1$. The main panel represents the case with $\eta \neq 0$. In the small panel, the results are presented for both $\eta=0$ and $\eta \neq 0$ cases with $g^{\prime}=0.6$.

effects fully taken into account $\left(\beta_{k i n}=\beta_{S, P}=1\right)$. As shown in Fig. 3, the interaction energy per nucleon depends on the Lorentz-Lorenz parameter rather weakly. Moreover, when $g^{\prime}$ decreases in such a way that it is taken to be the empirical $\left(g^{\prime}=0.6\right)$ or classic $\left(g^{\prime}=0.33\right)$, it is even more difficult to find the crystalline structure of the ground state. All curves in the main panel correspond to the full variational function, including parameter $\eta$. The small panel of Fig. 3 demonstrates the role of the variational parameter $\eta$, where $g^{\prime}=0.6$ is chosen.

The present results imply that the medium-modifications dramatically changes the picture of nuclear matter due to the following reasons: Firstly, the modifications of singlenucleon properties make the contribution of the quantum fluctuations increased, since the nucleon mass is decreased in the kinetic term (see eq. (9)). Secondly, the tensor part of the NN potential is suppressed in nuclear matter. As a result, its negative contribution to the total interaction energy is getting smaller.

It is interesting to study whether the medium modifications play a similar role, when the quantum fluctuations are suppressed. In order to see the effect of the medium modifications of hadron properties, we artificially vary the parameter $\beta_{k i n}$ to suppress the quantum fluctuations. The results are drawn in Fig. 4. The suppression of the quantum fluctuations leads obviously to lowering the interaction energy per nucleon. This effect is shown in the long-dashed and short-dashed curves in Fig. 4. When the quantum fluctuations become 


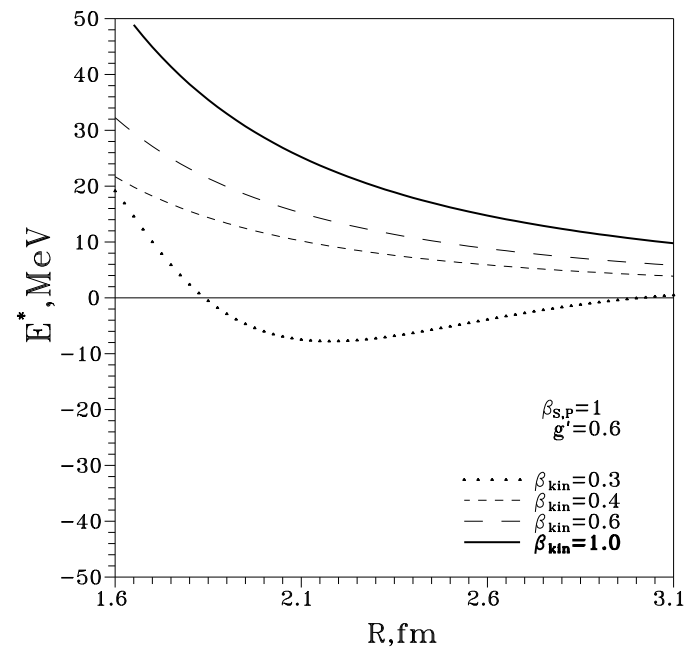

FIG. 4: The effect of the parameter $\beta_{k i n}$ on the interaction energy per nucleon (9). The medium modifications of hadron properties are fully taken into account $\left(\beta_{S, P}=1\right)$ and the Lorentz-Lorenz parameter is fixed to be $g^{\prime}=0.6$.

weak enough $\left(\beta_{\text {kin }} \approx 0.3\right)$, the ground-state energy of the system makes it possible for nuclear matter to be in the solid or condensed FCC configuration, as discussed already in Refs. [3, 4, 5, 11]. This is shown by the dotted curve in Fig. 4.

In the present work, we investigated the structure of nuclear matter in the framework of the medium-modified Skyrme model. We found that the medium modifications of the hadron properties and NN tensor potential are crucial to see whether the crystalline structure of nuclear matter exist or not. It turned out that the contribution of the medium modifications to the interaction energy per nucleon breaks a possible crystalline structure of the ground state of nuclear matter. We found that the present results are stable under the changes of numbers of variational parameters in the lattice-point nucleon trial functions.

However, one has to note that there might be a possibility that a condensed state at the normal nuclear-matter density could exist within the framework of the medium-modified Skyrme model according to the following reasons: Firstly, because of the contribution of the medium modifications to the interaction energy, a desirable minimum of nuclear matter seems to appear around the normal nuclear-matter density if quantum fluctuations are suppressed. The dotted curve in Fig. 4 indicates this possibility. However, we want to emphasize that without the medium modifications one should not conclude the existence of the crystalline structure of nuclear matter. Secondly, since it is well known that the binding of nuclear matter results from a strong cancellation between an attractive potential term and a repulsive kinetic one, the present conclusion may be changed if one can include the central attractive NN potential, which is in the present work neglected. For example, it is asserted in Ref. [19] that the scalar-isoscalar degrees of freedom are important. One can include the contribution of the scalar-isoscalar channel in the Skyrme model in a similar way as done for the pseudoscalar-isovector channel. For example, Refs. [13, 15, 20] showed that the attraction of the central NN potential is modified in nuclear matter in such a way that it is suppressed as the density increases. It is clear that due to these modifications the whole attractive potential containing the central and tensor parts will be lessened. However, if the scalar degrees of freedom are added in the medium-modified Skyrme model, the nucleon 
effective mass is less changed [15]. Consequently, the kinetic part on the interaction energy should be suppressed (quantum fluctuations will be suppressed). Therefore, it will be of great importance to understand the interplay between these terms in the interaction energy within the framework of the medium-modified Skyrme model, the scalar-isoscalar channel being considered.

\section{Acknowledgments}

The present work is supported by the Korea Research Foundation Grant (KRF-2003070-C00015). The work of UTY was financially supported by Pusan National University in program Post-Doc. 2004. MMM acknowledges the support of the Brain Pool program 2004 which makes it possible for him to visit Pusan National University.

[1] M.Kutschera, C.J. Pethick, D.G. Ravenhall, Phys. Rev. Lett. 53, 1041 (1984).

[2] I.R. Klebanov, Nucl. Phys. B 262, 133 (1985).

[3] N.K. Glendenning, Phys. Rev. C 34, 1072 (1986).

[4] E. Wüst, G.E. Brown, A.D. Jackson, Nucl. Phys. A 468, 450 (1987); A.S. Goldhaber and N.S. Manton, Phys. Lett. B 198, 231 (1987); M. Kugler and S. Shtrikman, Phys. Lett. B 208, 491 (1988); M. Kugler, S. Shtrikman, Phys. Rev. D 40, 3421 (1989).

[5] A.D. Jackson and J.J.M. Verbaarschot, Nucl. Phys. A 484, 419 (1988); L. Castillejo, P.S.J. Jones, A.D. Jackson, J.J.M. Verbaarschot, A. Jackson, Nucl. Phys. A 501, 801 (1989); H. Forkel, A.D. Jackson, M. Rho, C. Weiss, A. Wirzba and H. Bang, Nucl. Phys. A 504, 818 (1989); W.K. Baskerville, Nucl. Phys. A 596, 611 (1996).

[6] N.S. Manton, P.J. Ruback, Phys. Lett. B 181, 137 (1986); N.S. Manton, Commun. Math. Phys. 111, 469 (1987).

[7] A.D. Jackson, A. Wirzba, L. Castillejo, Phys. Lett. B 198, 315 (1987); A.D. Jackson, A. Wirzba, L. Castillejo, Nucl. Phys. A 486, 634 (1988); A.D. Jackson, C. Weiss, A. Wirzba and A. Lande, Nucl. Phys. A 494, 523 (1989).

[8] A.D. Jackson, N.S. Manton and A. Wirzba, Nucl. Phys. A 495, 499 (1989); A. Wirzba and H. Bang, Nucl. Phys. A 515, 571 (1990); A.D. Jackson, C. Weiss, A. Wirzba, Nucl. Phys. A 529, 741 (1991); A. Wirzba, arXiv:hep-ph/9211295.

[9] H.-J. Lee, B.-Y. Park, D.-P. Min, M. Rho, V. Vento, Nucl. Phys. A723, 427 (2003); H.-J. Lee, B.-Y. Park, M. Rho, V. Vento, Nucl. Phys. A726, 69 (2003).

[10] B. Y. Park, D. P. Min, M. Rho and V. Vento, Nucl. Phys. A 707, 381 (2002).

[11] D.I. Diakonov, A.D. Mirlin, Sov. J. Nucl. Phys. 47, 421 (1988) [Yad. Fiz. 47, 662 (1988)].

[12] T.S. Walhout, Nucl. Phys. A484, 397 (1988); T.S. Walhout, Nucl. Phys. A519, 816 (1990).

[13] J. W. Durso, H. C. Kim and J. Wambach, Phys. Lett. B 298 (1993) 267.

[14] A. Rakhimov, M. Musakhanov, F. Khanna, U. Yakhshiev, Phys. Rev. C58, 1738 (1998).

[15] A. Rakhimov, F. Khanna, U. Yakhshiev, M. Musakhanov, Nucl. Phys. A643, 383 (1999).

[16] U.T. Yakhshiev, M.M. Musakhanov, A.M. Rakhimov, Ulf-G. Meißner, A. Wirzba, Nucl. Phys. A700, 403 (2002); U.T. Yakhshiev, Ulf-G. Meißner, A. Wirzba, Eur. Phys. J. A16, 569 (2003).

[17] T. Ericson and W. Weise, Pions and Nuclei (Claredon, Oxford, 1988).

[18] G.S. Adkins, C.R. Nappy, E. Witten, Nucl. Phys. B228, 552 (1983). 
[19] B.D. Serot, J.D. Walecka, Adv. Nucl. Phys. 16, 1 (1986).

[20] U. Yakhshiev, U. Meißner, A. Wirzba, A. Rakhimov, M. Musakhanov, arXiv:nucl-th/0409002. 